\title{
Teste de Fluência na Leitura Silenciosa em Contexto para o Português Europeu: Estudo Preliminar de Validação com Estudantes Universitários
}

\author{
Silent Contextual Reading Fluency Test for European Portuguese: A Preliminary \\ Validation Study with University Students
}

\author{
Sandra Fernandes ${ }^{1}$, Luís Querido ${ }^{2}$, Marta Pereira ${ }^{3}$ e Miguel Domingues ${ }^{3}$
}

\begin{abstract}
Resumo
O objetivo do presente estudo foi desenvolver um instrumento para avaliação da Fluência na Leitura Silenciosa em Contexto (FLSC), com estudantes universitários portugueses. A sua construção foi inspirada num teste de FLSC dos EUA. Inclui duas formas equivalentes. Cada uma contém um texto escrito em letras maiúsculas, sem pontuação nem espaços entre palavras. Os estudantes tinham de desenhar um traço separador entre o maior número de palavras possível, numa condição de tempo limitado. O teste foi administrado a um total de 50 estudantes universitários, em pequenos grupos (entre 5 e 10 participantes). Foi obtida uma adequada fiabilidade entre as formas e uma boa validade concorrente, com um teste de compreensão em leitura silenciosa. $\mathrm{O}$ teste revelou-se uma medida rápida, precisa e de baixo custo para a avaliação da FLSC, agora disponível no Português Europeu, útil para a investigação e a clínica. Limitações e direções futuras são discutidas.
\end{abstract}

Palavras-chave: fluência, fluência na leitura silenciosa, leitura em contexto, compreensão em leitura

\begin{abstract}
The aim of this study was to develop an instrument to assess contextualized silent reading fluency in Portuguese university students. The test construction was inspired on a U.S. Test of Silent Contextual Reading Fluency. It includes two equivalent forms. Each of them contains a text printed in uppercase without punctuation and spaces between words. Students had to draw a line between as many individual words as possible in a time limited condition. The test was administered in small groups (5 to 10 participants) to a total of 50 Portuguese university students. An appropriate reliability for the two forms, and a good concurrent validity with a silent reading comprehension test was observed. Therefore, the test proved to be a quick, accurate and cost-efficient measure of silent reading ability now available for European Portuguese, useful for research and/or clinical purposes. Limitations and future research directions are discussed.
\end{abstract}

Keywords: fluency, silent reading fluency, contextual reading

Agradecemos a todos os estudantes do $1^{\circ}$ ano do Mestrado Integrado em Psicologia da Faculdade de Psicologia da Universidade de Lisboa que participaram no estudo.

\footnotetext{
${ }^{1}$ Doutorada em Psicologia Cognitiva. Faculdade de Psicologia, Universidade de Lisboa. Professora Auxiliar. Endereço: Faculdade de Psicologia, Alameda da Universidade, 1649-013 Lisboa, Portugal. E-mail: sfernandes@ psicologia.ulisboa.pt

${ }^{2}$ Doutorado em Psicologia Cognitiva. Faculdade de Psicologia, Universidade de Lisboa. Neuropsicólogo no Serviço à Comunidade da Faculdade de Psicologia da Universidade de Lisboa. Endereço: Serviço à Comunidade da Faculdade de Psicologia, Alameda da Universidade, 1649-013 Lisboa, Portugal.

${ }^{3}$ Licenciada/o em Ciências Psicológicas. Faculdade de Psicologia, Universidade de Lisboa. Estudante do Mestrado Integrado em Psicologia. Endereço: Faculdade de Psicologia, Alameda da Universidade, 1649-013 Lisboa, Portugal.
} 


\section{Introdução}

A alfabetização pode ser encarada como um processo que não tem um início e um fim definidos, acontecendo ao longo de todo o ciclo de vida (Piacente, 2012). No entanto, até à data, a maior parte dos estudos acerca do desenvolvimento da leitura, e em particular da fluência em leitura, centram-se nos estudantes do ensino básico (e.g., Rasinski, Padak, Linek, \& Sturtevant, 1994), com especial enfoque no $1^{\circ}$ ciclo, negligenciando o papel da fluência ao nível dos ensinos secundário e superior (e.g., Barth, Catts, \& Anthony, 2009). Contudo, em níveis de escolaridade mais avançados, como o ensino universitário, é crítico ser-se um leitor fluente, para fazer face às exigências de leitura características desta fase da aprendizagem.

Apesar dos dados da Organização para a Cooperação e Desenvolvimento Económico de 2015 (OECD, 2016 / PISA, 2015), no que se refere à proficiência na leitura de estudantes entre os 15 e os 16 anos, serem encorajadores para Portugal, uma vez que se encontra entre os países com maior evolução desde 2000, apenas $7.5 \%$ dos estudantes portugueses se situam nos níveis associados às tarefas de maior complexidade e exigência. Além disso, é de sublinhar que 17,5\% dos estudantes portugueses apresentam um desempenho igual ou inferior ao nível 2 (em 7) de proficiência na leitura (OECD, 2016/PISA, 2015). Assim, como apontado por Fernandes, Simões, Querido e Verhaeghe (2015), com referência aos resultados do PISA (2009), muitos adolescentes continuam a "apresentar dificuldades no cumprimento das exigências académicas, em resultado de um nível deficiente de mestria nas habilidades de leitura" (p.114).

Os leitores proficientes respondem ao material escrito com velocidade, precisão e ausência de esforço, reproduzindo um texto escrito em linguagem oral (e.g., Ehri, 2005). De acordo com Fuchs, Fuchs, Hosp e Jenkins (2001), a fluência na leitura oral pode servir como um indicador da competência geral de leitura, representando o desempenho multifacetado e complexo em resultado de um conjunto coordenado de processos (e.g., conversão grafema-fonema, acesso lexical automático, estabelecimento de ligações de significado dentro e entre frases e relação entre o significado do texto e o conhecimento anterior). Assim, a fluência na leitura oral reflete uma "orquestração complexa que pode ser usada de um modo elegante e fiável para caracterizar a leitura proficiente" (Fuchs et al., 2001, p.240)

Neste sentido, a fluência na leitura oral é, com mais frequência, expressa através do número de palavras lidas corretamente em voz alta, por minuto, quer isoladamente quer num texto, ou em contexto (Jenkins, Fuchs, van den Broek, Espin, \& Deno, 2003; Fuchs et al., 2001). No entanto, é amplamente reconhecido que a fluência em leitura engloba três dimensões: precisão, velocidade e prosódia (e.g., Hudson, Pullen, Lane, \& Torgesen, 2009). No presente estudo não é nosso objetivo estudar esta última dimensão.

Segundo Laberge e Samuels (1974), é a fluência em leitura que facilita em primeiro lugar a compreensão. Quando a identificação das palavras se torna mais rápida e mais automática, ou seja, mais fluente, libertam-se recursos cognitivos para os processos de compreensão do texto (Laberge \& Samuels, 1974; Perfetti, 1985). Assim, a falta de automaticidade na leitura, da habilidade de reconhecer as palavras rapidamente, prestando pouca atenção à palavra em si mesma (ou seja, acedendo automaticamente às representações lexicais), compromete a construção de significado e consequentemente a compreensão na leitura de textos (e.g., Perfetti, 1985), além de causar, em muitos estudantes, problemas de carácter psicológico, como resultado do insucesso na prossecução de estudos superiores. Portanto, e de acordo com Rasinski e colaboradores (Rasinski, 2006; Rasinski et al., 2005) deve ser dada alguma atenção à fluência em leitura no ensino secundário e em anos posteriores, bem como à necessidade de desenvolver mais investigação nestes períodos da aprendizagem. De qualquer forma, é de notar que um número crescente de estudos tem sido conduzido no que se refere à fluência em leitura desde o início do século (e.g., Chard, Vaughn, \& Tyler, 2002; Hudson et al., 2009), desde a impulsionadora informação apresentada pelo National Reading Panel em 2000, que sublinhou a importância da instrução e eficácia na fluência em leitura. Também em Português tem sido dedicada alguma investigação acerca da fluência em leitura 
durante o ensino básico (e.g., Fernandes, Querido, Verhaeghe, Marques, \& Araújo, 2017; Fernandes, Querido, Verhaeghe, \& Araújo, 2018) e secundário (e.g., Fernandes et al., 2015). Este interesse no estudo da fluência em leitura surge fundamentalmente por esta ser reconhecidamente considerada como um importante indicador da habilidade geral de leitura (e.g., Fuchs et al., 2001) e estar amplamente relacionada com a compreensão em leitura (Pikulski \& Chard, 2005; Rasinski, 2009).

De um modo geral, nos estudos empíricos acerca da fluência em leitura, e em particular nos níveis iniciais da aprendizagem, as medidas são de leitura em voz alta (e.g., Fernandes et al., 2017, 2018; Fuchs et al., 2001), o que faz sentido, considerando que estas medidas trazem benefícios para os leitores principiantes uma vez que refletem o modo como estão habituados a ler em contexto de aprendizagem, por exemplo. De facto, as crianças no início da aprendizagem em leitura são expostas primeiramente à leitura em voz alta por parte dos adultos e vão praticando a leitura em voz alta com o apoio de um leitor hábil (Chall, 1996), o/a professor/a na maior parte dos casos. Por volta do quarto ano de escolaridade é expectável uma efetiva transição para a leitura silenciosa sem a necessidade do apoio da automonitorização típica da leitura oral (Hiebert, Samuels, \& Rasinski, 2012). No ensino posterior ao primeiro ciclo do ensino básico ( $4^{\circ}$ ano) a maior parte das atividades de leitura são realizadas silenciosamente. Vários estudos têm mostrado que a fluência na leitura oral e a fluência na leitura silenciosa estão relacionadas entre si (e.g., Kim, Wagner, \& Foster, 2011, Berninger et al., 2010; Denton et al., 2011, respetivamente com estudantes do $1^{\circ}$ ano, do $4^{\circ}$ e do ensino secundário) e apresentam correlações significativas com a compreensão em leitura (e.g., Denton et al., 2011; Paige, Rasinski, \& MagpuriLavell, 2012; Yildirim \& Ates, 2012).

Assim, a avaliação da fluência na leitura silenciosa reveste-se de utilidade para a identificação de estudantes com eventuais dificuldades de leitura e para a avaliação dos seus níveis de progressão nesta habilidade e na compreensão em leitura, em particular em níveis mais avançados da escolaridade. Neste sentido, o objetivo deste estudo foi desenvolver, para o
Português Europeu, um primeiro teste de fluência na leitura silenciosa em contexto, inspirado no Test of Silent Contextual Reading Fluency de Hammill, Wiederholt e Allen (2006) e levar a cabo um estudo preliminar de fiabilidade e validade do mesmo, com estudantes universitários portugueses.

De facto, para a língua Inglesa, está há mais de uma década, disponível uma medida de fluência na leitura silenciosa de aplicação coletiva, o Test of Silent Contextual Reading Fluency (TOSCRF; Hammill et al., 2006), que foi desenvolvido para ultrapassar em particular a crítica relativa ao elevado tempo de aplicação (individual) e cotação subjacentes dos testes tradicionais de fluência na leitura oral. $\mathrm{O}$ referido teste (TOSCRF) exige que os estudantes assinalem a fronteira entre palavras num texto, num tempo limitado.

Para além destas vantagens, inerentes a um teste desta natureza, o Teste que nos propusemos a desenvolver e validar para o Português Europeu, será a primeira medida de fluência na leitura silenciosa em contexto disponível na nossa língua, com dados de referência para estudantes universitários. Do nosso conhecimento, até à data, apenas um teste "O Rei" (Carvalho \& Pereira, 2009) de fluência na leitura de texto está disponível para o português europeu e é dedicado à avaliação da leitura em voz alta (fluência e precisão), do $1^{\circ}$ ano ao $6^{\circ}$ ano de escolaridade.

O propósito do presente estudo prende-se, então, com o facto de a análise da fluência na leitura silenciosa de textos ser considerada a melhor forma de avaliar a leitura em leitores hábeis (e.g., Gagliano et al., 2015), enquanto a fluência na leitura em voz alta é considerada de maior utilidade para a avaliação da competência geral da leitura de estudantes em níveis mais iniciais de escolaridade (e.g., Fuchs et al., 2001). Além disso, existem poucos dados empíricos que apoiem o uso da fluência na leitura oral como um indicador de diagnóstico de estudantes mais velhos e adultos com suspeita de dificuldades específicas de aprendizagem (Gagliano et al., 2015).

Como referido anteriormente, o teste que desenvolvemos teve como ponto de partida os princípios e características do TOSCRF (Hammill et al., 2006), disponível para a língua inglesa, 
reconhecendo a necessidade da existência de ferramentas válidas que possibilitem aos técnicos (professores, psicólogos, entre outros) a identificação, o diagnóstico e a monitorização do progresso da habilidade de leitura de estudantes além do ensino secundário.

É sabido que a fluência na leitura silenciosa em contexto é um importante e forte preditor da compreensão em leitura, quer com estudantes do ensino básico (e.g., Berninger et al., 2010, no $4^{\circ}$ ano; Denton et al., 2011, do $6^{\circ}$ ao $8^{\circ}$ ano), quer com estudantes do ensino secundário e posterior (e.g., Seok \& DaCosta, 2014), tendo sido parte destes estudos empíricos (e.g., Denton et al., 2011; Seok \& DaCosta, 2014) levados a cabo com o TOSCRF (Hammill et al., 2006).

A partir dos 17/18 anos, com a entrada no Ensino Superior, espera-se que os estudantes, comecem a sintetizar a multiplicidade de pontos de vista presentes nos textos para determinar a sua própria perspetiva acerca de um determinado assunto. Esta habilidade de sintetizar diversos pontos de vista é fundamental para o desenvolvimento de um leitor crítico. Neste nível (Chall, 1996), a leitura é considerada construtiva, ou seja, o leitor constrói conhecimento e compreensão a partir da leitura do que outros escreveram. De acordo com esta teoria (Chall, 1996) só um leitor fluente alcançará este estádio (para uma revisão da teoria, ver Kuhn \& Stahl, 2003). Como é do nosso conhecimento, muitos dos estudantes que entram no ensino superior apresentam dificuldades na leitura, quer em Portugal, a julgar pelos resultados de alunos adolescentes (OCDE, 2017/ PISA 2015), e pelos baixos resultados obtidos nos exames nacionais de Português no $12^{\circ}$ ano, quer noutros países (e.g., Trainin \& Swanson, 2005, na língua inglesa). Além disso, é bastante provável que haja um grupo de estudantes, que alcança o ensino superior, com dificuldades de aprendizagem (incluindo dislexia), sem ter sido formalmente diagnosticado (Tops, Callens, Lammertyn, Van Hees, \& Brysbaert, 2012). De facto, no Reino Unido, por exemplo, cerca de $3,2 \%$ dos estudantes do ensino superior têm dislexia (Warmington, Stothard, \& Snowling, 2013), mas este número é considerado subestimado, pois segundo Warmington et al. (2013), $40 \%$ a $43 \%$ dos estudantes com dislexia no ensino superior não estarão diagnosticados e, por isso, permanecem até ao último ano do percurso académico universitário por identificar e apoiar. Em Portugal estão em falta dados relativos à incidência da dislexia no ensino superior, uma vez que, apenas recentemente, dispomos de um teste de screening (TIL-1min: Fernandes, Araújo, Sucena, Reis, \& Castro, 2017) e não dispomos de testes de diagnóstico destas dificuldades para este nível de ensino. A escassez de medidas precisas de avaliação da leitura nesta faixa etária, em Portugal, precisa de ser colmatada, de modo a poder-se monitorizar o progresso da habilidade de leitura nas suas vertentes da automaticidade e compreensão. É nosso objetivo contribuir para o preenchimento desta lacuna em Portugal. O TOSCRF (Hammill et al., 2006) exige, quer o acesso automático à representação lexical das palavras, quer a habilidade de compreensão em leitura: "O TOSCRF engloba muitas das habilidades essenciais de compreensão, incluindo a identificação, o significado (vocabulário) e a construção (morfologia) de palavras, a estrutura das frases (sintaxe) e relações de significado (compreensão)" (Allen et al., 2011, p.2). Neste sentido, inspirados por este teste (TOSCRF, Hammill et al., 2006), desenvolvemos o Teste de Fluência na Leitura Silenciosa em Contexto para o Português Europeu e apresentamos aqui dados preliminares da sua validação.

Foram desenhadas duas formas equivalentes do teste, de modo a permitir a monitorização do progresso da habilidade de leitura. Cada uma das formas consistia na apresentação de um texto no qual todos os espaços e pontuação foram removidos. A tarefa, realizada coletivamente, requeria a identificação do maior número possível de palavras através do desenho de linhas separadoras, num tempo limitado. Este procedimento torna $\mathrm{o}$ teste de fácil e rápida aplicação.

É nosso objetivo divulgar dados de referência de estudantes universitários no que se refere à fluência na leitura silenciosa e apresentar resultados preliminares de validade concorrente do Teste de Fluência na Leitura Silenciosa em Contexto, que foi desenvolvido através da análise da sua relação com o teste de compreensão em Leitura silenciosa de frases TIL-1min (Fernandes, et al.,2017) e do Questionário de História de 
Leitura (Lefly \& Pennington, 2000; Versão portuguesa de Alves \& Castro, 2005). Foram estes os instrumentos escolhidos para o estudo da validade do Teste de Fluência na Leitura Silenciosa em Contexto (de texto) atendendo a que, 1) até à data, do nosso conhecimento, o teste TIL-1min é o único disponível para a avaliação do mesmo construto (leitura silenciosa em contexto), neste caso a leitura com compreensão de frases, de adultos portugueses; e a que 2) alguns estudos sugerem que medidas objetivas de leitura se correlacionam com medidas de autorrelato relativas a esta habilidade (Nergård-Nilssen \& Hulme, 2014; Tamboer \& Vorst, 2015). Neste sentido, são esperadas correlações entre os desempenhos nestes instrumentos de medida.

\section{Método}

\section{Participantes}

Neste estudo participou uma amostra, obtida por conveniência, de 50 estudantes universitários, do $1^{\circ}$ ano do Mestrado Integrado em Psicologia da instituição de afiliação dos autores.

Dos 50 participantes, 43 eram do sexo feminino e 7 do sexo masculino, com uma média de idades de 20.78 (desvio-padrão: 5.58).

Os critérios de inclusão na amostra foram os seguintes: possuir o Português Europeu como língua materna, não sendo bilingues; não ter problemas auditivos e/ou visuais não corrigidos, não ter dificuldades articulatórias (e.g., gaguez), défice de atenção e concentração ou hiperatividade, e não estar a tomar medicação psiquiátrica ou neurológica.

$\mathrm{O}$ presente estudo fez parte de um projeto mais alargado no âmbito do desenvolvimento da leitura, aprovado pela Comissão de Ética e Deontologia da instituição de afiliação dos autores. Os estudantes participaram com consentimento informado.

\section{Instrumentos}

\section{Teste de Fluência na Leitura Silenciosa em Contexto}

O Teste de Fluência na Leitura Silenciosa em Contexto consistiu na apresentação de um texto (neste caso apresentado em cadeias de palavras) em letra maiúscula, no qual todos os espaços e pontuação foram removidos. Foram desenhadas duas formas equivalentes do teste. Os participantes foram instruídos para ler da esquerda para a direita, linha a linha como habitualmente aquando da leitura de um texto. O teste começou com um exemplo seguido de três frases que constituíram os exercícios de prática a realizar num minuto. Após estes exercícios foi dado feedback corretivo através da leitura correta de cada uma das frases. Concluída a prática, passouse ao teste propriamente dito. As duas formas do teste foram apresentadas consecutivamente. Cada forma consistiu num texto (171 palavras em 9 frases na forma A e 167 palavras em 9 frases na forma B). Os textos foram construídos e adaptados para o Português Europeu a partir da obra de John Guy (2005). De acordo com a Flesch Reading Ease Formula (Fernandez-Huerta, 1959) para a língua Espanhola, os textos das duas formas de teste são de legibilidade equivalente (11.20 e 9.67, respetivamente para a Forma A e B), adequados ao nível de ensino universitário. Os participantes tinham dois minutos para desenhar linhas separadoras entre o máximo de palavras possível. Cada forma foi aplicável em cerca de 5 minutos (incluindo exercícios de prática e instruções). A ordem das formas foi contrabalançada entre os grupos. O desempenho dos participantes foi avaliado pelo número de palavras corretamente separadas nos dois minutos de teste.

\section{Teste de Compreensão em Leitura (Teste de Idade} de Leitura, TIL - 1min, Fernandes et al., 2017)

O TIL-1min (Fernandes et al., 2017) é um teste que avalia a compreensão em leitura silenciosa nos adultos, através do completamento de frases, a partir de cinco alternativas de escolha, com tempo limitado a 1 minuto. $\mathrm{O}$ teste consistiu na apresentação de quatro exercícios de prática sem tempo limite, com feedback corretivo. Após estes primeiros exercícios, os participantes tinham 1 minuto para completar o maior número de frases possível de um conjunto de 36 . O número de frases completadas corretamente permitiu a avaliação do desempenho dos participantes. O estudo de validade do teste (Fernandes et al., 2017) refere boas propriedades psicométricas 
(coeficientes de teste-reteste $>.70$ e consistência interna de .78).

Questionário de História de Leitura de Lefly e Pennington (2000) (Versão Portuguesa de Alves \& Castro, 2003)

A versão portuguesa do Questionário de História de Leitura (Alves \& Castro, 2003) é constituída por um conjunto de 25 itens de resposta com formato Likert (escala 0 - 4) e 4 com formato de resposta informativa, tratando-se de uma medida de autorrelato de dificuldades de leitura e escrita durante a escola primária (e.g., "Sentiu dificuldades a aprender a ler na escola primária?"; "Na escola primária, foi difícil aprender a escrever o português corretamente (sem erros ortográficos)?"). Os participantes assinalaram com um círculo o número da escala que correspondia à sua resposta. Quanto maior o valor total obtido maior a dificuldade, numa pontuação máxima de 100 pontos. O índice de consistência interna (alfa de Cronbach) obtido na amostra do presente estudo foi de .75 .

\section{Procedimento}

As aplicações decorreram em sessões coletivas, em pequenos grupos (entre 5 e 10 participantes), com uma duração total de aproximadamente 20 minutos. A ordem de aplicação dos instrumentos foi: 1) Teste de Fluência na Leitura Silenciosa em Contexto (formas A e B com apresentação alternada entre grupos); 2) TIL-1min (Fernandes et al., 2017); e 3) Questionário de História de Leitura (Alves \& Castro, 2003).

\section{Resultados}

\section{Coeficiente de Confiança entre a Forma A e B do teste e Estatística Descritiva}

Observou-se que os dados apresentavam uma distribuição normal através do teste Shapiro-Wilk ( $p>.10$ para ambas as formas do Teste de Fluência na Leitura Silenciosa em Contexto, para o TIL1min e para o Questionário de História de Leitura).

No Quadro 1 apresenta-se o Coeficiente de Confiança e a Estatística Descritiva entre as formas do Teste de Fluência na Leitura Silenciosa em Contexto. A magnitude do coeficiente sugere uma boa confiança entre as duas formas do teste.
Quadro 1. Estatística Descritiva e Coeficiente de Confiança entre Formas do Teste de Fluência na Leitura Silenciosa em Contexto

\begin{tabular}{lccccc}
\hline & \multicolumn{2}{c}{ Forma A } & \multicolumn{3}{c}{ Forma B } \\
\hline & $M$ & $D P$ & $M$ & $D P$ & $r$ \\
\hline $\begin{array}{l}\text { Teste de } \\
\text { Fluência na }\end{array}$ & & & & & \\
$\begin{array}{l}\text { Leitura } \\
\text { Silenciosa } \\
\text { em }\end{array}$ & 96.10 & 15.40 & 92.30 & 15.55 & $.74^{* *}$ \\
Contexto & & & & & \\
\hline
\end{tabular}

Nota. Número de palavras corretas em 2 minutos; ** $p<.001$

O desempenho médio dos participantes na realização da forma $\mathrm{A}$ foi de $96.10(\mathrm{DP}=15.40)$ palavras corretas em dois minutos e de 92.30 $(\mathrm{DP}=15.55)$ na realização da Forma $\mathrm{B}$ (ver Quadro 1).

Para obter um panorama mais completo do desempenho em leitura silenciosa no Teste de Fluência, determinámos os percentis associados a diferentes níveis de fluência (ver Quadro 2).

Quadro 2. Percentis associados a diferentes níveis de desempenho (número de palavras identificadas corretamente em dois minutos) obtidos no Teste de Fluência na Leitura Silenciosa em Contexto (Formas A e B)

\begin{tabular}{lcc}
\hline & \multicolumn{2}{c}{ Número de palavras corretas } \\
\hline Percentís & Forma A & Forma B \\
\hline 5 & 69.85 & 59.10 \\
15 & 80.00 & 72.00 \\
25 & 85.50 & 87.00 \\
50 & 94.50 & 94.00 \\
75 & 109.25 & 102.50 \\
85 & 113.00 & 108.35 \\
95 & 120.90 & 113.45 \\
\hline
\end{tabular}

Atendendo aos valores obtidos correspondentes ao percentil 25, em ambas as Formas (85.50 e 87.00, respetivamente para a Forma A e B) sugere-se que 85 palavras identificadas corretamente, em qualquer das Formas do teste, seja um ponto de corte indiciador de eventuais dificuldades de leitura.

\section{Validade concorrente}

Considerando que o Teste de Fluência na Leitura Silenciosa em Contexto foi desenvolvido 
para avaliar a leitura em contexto, dever-se-á correlacionar significativamente com outras medidas de leitura e, em particular de compreensão em leitura. Neste sentido, examinámos a validade concorrente do Teste de Fluência na Leitura Silenciosa em Contexto (Formas A e B) determinando a sua correlação com o Teste de Compreensão em Leitura silenciosa (TIL-1min) e um Questionário de História de Leitura. Como se pode observar no Quadro 3, ambas as Formas do Teste de Fluência na Leitura Silenciosa em Contexto apresentam valores de correlação moderados a altos com a medida de compreensão em leitura ( $r=.46$ e $r=.55$, respetivamente para a Forma A e B), sendo de referir que a o valor de correlação da Forma $\mathrm{B}$ foi ligeiramente superior ao da Forma A.

O Teste de Fluência na Leitura Silenciosa em Contexto não apresentou correlações significativas com o Questionário de História de Leitura.

Quadro 3. Validade concorrente: Matriz de correlações entre o Teste de Fluência na Leitura Silenciosa em Contexto (Formas A e B), o Teste de Compreensão em Leitura (TIL-1 min) e o Questionário de História de Leitura (Alves \& Castro, 2005)

\begin{tabular}{|c|c|c|c|c|c|}
\hline & & 1 & 2 & 3 & 4 \\
\hline 1 & $\begin{array}{l}\text { Teste de Fluência na } \\
\text { Leitura Silenciosa em } \\
\text { Contexto - Forma A }\end{array}$ & 1 & $.74 * *$ & $.46^{* *}$ & -.10 \\
\hline 2 & $\begin{array}{l}\text { Teste de Fluência na } \\
\text { Leitura Silenciosa em } \\
\text { Contexto - Forma B }\end{array}$ & & 1 & $.55^{* *}$ & -.16 \\
\hline 3 & $\begin{array}{l}\text { Teste de Compreensão em } \\
\text { Leitura (TIL-1 min) }\end{array}$ & & & 1 & -.12 \\
\hline 4 & $\begin{array}{l}\text { Questionário de História de } \\
\text { Leitura (Alves \& Castro, } \\
\text { 2005) }\end{array}$ & & & & 1 \\
\hline
\end{tabular}

\section{Discussão}

Procurar estabelecer a validade de instrumentos de avaliação é de extrema importância, uma vez que a avaliação deve fundamentar-se em dados de estudos empíricos obtidos com métodos adequados que revelem características psicométricas fiáveis. Em Portugal, não se encontram disponíveis instrumentos de medida de fluência em leitura para alunos do ensino superior. Foi nosso objetivo, com o presente estudo, contribuir para começar a colmatar esta lacuna no que se refere à avaliação da fluência na leitura silenciosa de estudantes mais velhos.

Como referido anteriormente, a maior parte dos estudos sobre a leitura, e em particular sobre fluência em leitura, centra-se nos estudantes do ensino básico, negligenciando-se os níveis secundário e universitário. No entanto, ler com fluência nos níveis posteriores de ensino/aprendizagem é requerido e fundamental para o sucesso académico. Além disso, as medidas de fluência são geralmente de leitura em voz alta, quando a maior parte das atividades de leitura, em particular dos leitores hábeis, são realizadas silenciosamente.

Posto isto, foi nosso propósito desenvolver, para o Português Europeu, um teste de fluência na leitura silenciosa em contexto e apresentar dados preliminares das suas propriedades psicométricas. O Test of Silent Contextual Reading Fluency (TOSCRF) de Hammill et al. (2006) foi inspirador para levar a cabo este primeiro estudo exploratório de fiabilidade e validade do mesmo, com estudantes universitários portugueses. Foram desenhadas duas formas equivalentes do teste. Cada uma das formas consistia na apresentação de um texto no qual todos os espaços e pontuação foram removidos. Os participantes tinham dois minutos para identificar as fronteiras do máximo de palavras possível, marcando-as com um traço "|" separador. O teste foi aplicado coletivamente, em pequenos grupos, a 50 estudantes universitários. Cada forma é aplicável em cerca de 5 minutos (incluindo instruções).

Os resultados obtidos revelaram um adequado coeficiente de confiança entre as duas formas A e B (índice de confiança de .74). Este resultado é comparável ao referido no estudo de Bell, McCallum, Kirk, Fuller e McCana-Bowling (2007) para o TOSCRF (Hammill et al., 2006) com adolescentes (índice de confiança de .70) e ligeiramente inferior aos obtidos por Allen e Rogers (2008), neste caso com índices de confiança de .82 a .87 entre várias formas, com estudantes dos 7 aos 18 anos.

A automaticidade na leitura foi determinada pelo número de palavras identificadas corretamente pelos estudantes em dois minutos. $\mathrm{O}$ 
desempenho dos estudantes foi de 96.10 palavras corretamente identificadas para a forma $\mathrm{A}$ e de 92.30 para a forma B. Estes desempenhos de estudantes portugueses do primeiro ano do ensino universitário são superiores aos observados com estudantes adolescentes (com média de idade de 13 anos) dos Estados Unidos (Bell et al., 2007). Neste caso, apresentaram um desempenho de 92.85 (Forma A do TOSCRF) e 101.14 (Forma B do TOSCRF) palavras corretamente identificadas em três minutos, o que corresponderia a 61.9 e 67.43, respetivamente para a Forma A e B, em dois minutos de tempo limite. A diferença de desempenho entre os dois grupos etários é notória, sugerindo uma progressão ao longo do desenvolvimento caracterizada nos modelos de desenvolvimento da leitura (e.g., Chall, 1996), embora devamos ter em atenção as diferenças no grau de consistência entre as ortografias inglesa e portuguesa, sendo a última menos inconsistente. Esta aparente evolução (trata-se de duas línguas distintas), é concordante com dados obtidos no Português Europeu (Fernandes et al., 2015), embora numa tarefa de fluência na leitura oral de texto, que revelam uma diferença no desempenho de estudantes do oitavo ano (156.6 palavras por minuto num texto; $\mathrm{DP}=23.7)$ e décimo ano de escolaridade (181.4; $\mathrm{DP}=27.3)$, com os últimos a revelarem um maior número de palavras lidas corretamente em voz alta num texto, relativamente aos primeiros. Note-se que, como referido anteriormente, os dois tipos de fluência na leitura de texto, oral e silenciosa, estão relacionados (e.g., Denton et al., 2011, com estudantes do ensino secundário; Seok \& DaCosta, 2014, com estudantes do ensino secundário e posterior).

A validade concorrente do Teste de Fluência na Leitura Silenciosa em Contexto foi avaliada através da correlação com um teste de compreensão em leitura (de frases) silenciosa (TIL-1min; Fernandes et al., 2017) e o questionário de história de leitura de Alves e Castro (2005).

Observámos uma boa validade concorrente (correlação moderada a alta) de ambas as formas ( $r=.46$ e $r=.55$, respetivamente para a Forma A e B) do teste com o teste de compreensão em leitura (de frases) silenciosa TIL-1min (ver Quadro 3). Os valores observados aproximam-se dos valores de correlação entre o TOSCRF e outras medidas de compreensão em leitura referidos por Allen e Rogers (2008) e por Allen, Hammill, Rogers e Jagielko (2010) em estudos de validade com população norte-americana. Tal como nestes estudos, os valores que observámos, de acordo com Hopkins (2002), são indicadores de uma relação moderada a forte com esta medida de validade concorrente, no mesmo domínio da leitura. Estes dados conferem robustez ao instrumento desenvolvido, enquanto medida de compreensão em leitura. Esta relação é de particular importância, uma vez que em níveis mais avançados de ensino/aprendizagem, os estudantes apresentam muitas vezes falta de automaticidade no reconhecimento das palavras, o principal componente da fluência em leitura, apresentando assim dificuldades na construção de sentido do material lido, ou seja, na compreensão do que leem (e.g., Paige, Rasinski, \& MagpuriLavell, 2012). E como é sabido, a automaticidade na leitura é fundamental para que o leitor liberte recursos que possa dedicar aos mecanismos de compreensão em leitura (Laberge \& Samuels, 1974; Perfetti, 1985). Além disso, como já referido, tem-se observado que a fluência na leitura silenciosa em contexto é um forte preditor da compreensão em leitura quer em estudantes do ensino básico (e.g., Denton et al., 2011, com alunos do $6^{\circ}$ ao $8^{\circ}$ ano) quer em estudantes de níveis mais avançados, secundário e superior (e.g., Seok \& DaCosta, 2014).

Ainda no que se refere à validade concorrente, o Teste de Fluência na Leitura Silenciosa em Contexto embora não tenha apresentado correlações significativas com uma medida subjetiva de leitura - o Questionário de História de Leitura - estas correlações são negativas, o que vai no sentido esperado. Ou seja, valores elevados no Questionário de História de Leitura, reveladores de dificuldade, relacionam-se com baixos desempenhos no Teste de Fluência na Leitura Silenciosa em Contexto e no Teste de Compreensão em Leitura (TIL-1min), ambos, medidas diretas da habilidade de leitura. É de referir que no estudo de validação do teste TIL1 min (Fernandes et al., 2017) também não foram observadas correlações significativas entre este teste e o mesmo questionário, numa amostra de estudantes universitários, considerados leitores 
típicos. No entanto, quando a amostra incluía leitores disléxicos, a relação encontrada foi significativa, revelando a possibilidade de diferenciação entre disléxicos e não disléxicos através do Questionário de História de Leitura. As autoras sugerem que os estudantes do ensino superior estão altamente expostos a material escrito e, por isso, têm oportunidade de estar conscientes das suas habilidades de leitura (Fernandes et al., 2017).

Os níveis de fluência na leitura silenciosa em contexto associados a diferentes percentis (ver Quadro 2) foram também determinados para ambas as formas do teste. Estabelecer um ponto de corte (85 palavras em 2 minutos) no desempenho do Teste de Fluência na Leitura Silenciosa em Contexto é particularmente importante para o diagnóstico e intervenção em dificuldades de leitura que podem afetar a compreensão em leitura e o desempenho académico global dos estudantes. No entanto, este indicador deve ser tomado como um ponto de referência que aponta para eventuais dificuldades, que deve ser complementado com uma avaliação analítica dos processos de leitura (e.g., com tarefas de consciência fonológica, leitura de palavras e de pseudopalavras) eventualmente em défice, que permitirá chegar, ou não, a um diagnóstico preciso de dificuldades.

\section{Limitações e investigação futura}

Estamos conscientes de que o presente trabalho constitui um estudo preliminar de validação do Teste de Fluência na Leitura Silenciosa em Contexto. O número de participantes, a distribuição de géneros e o restrito contexto académico dos mesmos constituem limitações do presente estudo, na medida em que reduz a variabilidade e representatividade dos resultados. Trabalhos futuros devem incluir jovens estudantes de uma maior variedade de contextos e áreas de estudo. A inclusão de diferentes grupos etários anteriores ao ensino universitário levaria a uma maior variabilidade de desempenhos, uma vez que o ensino superior constitui uma seleção de estudantes.

Embora o número de participantes nos tenha permitido levar a cabo algumas importantes e impulsionadoras análises estatísticas preliminares, sentimos que se trata de um número modesto.
Amostras com uma maior dimensão (e mais representativas) permitir-nos-iam levar a cabo análises mais complexas (e.g., curvas ROC; estudos de predição).

A precisão do teste deverá ser, no futuro, explorada com o procedimento teste-reteste.

A administração de testes adicionais (e.g., fluência na leitura oral de palavras) complementaria os resultados (e.g., contribuindo para a validade concorrente do teste em estudo) e a discussão dos mesmos. Contudo, não temos disponíveis em Portugal outros testes de leitura normativos para a população em estudo.

A utilidade clínica do teste deverá ser também alvo de estudo, contemplando amostras de leitores em dificuldade.

Atendendo às limitações enunciadas, os níveis de fluência na leitura silenciosa em contexto associados a diferentes percentis devem ser analisados com cautela e tomados apenas como indicadores, não dispensando uma avaliação analítica dos processos de leitura.

Apesar destes constrangimentos, os resultados obtidos revelaram uma adequada fiabilidade entre as formas do teste e boa validade concorrente (com um teste de compreensão em leitura), o que torna este novo instrumento, de aplicação rápida, de confiança, quer como um teste de screening, quer como um instrumento de avaliação da habilidade de leitura a incluir num protocolo de avaliação. Tratando-se de um teste que implica muitas das habilidades de leitura, desde a identificação de palavras, morfologia, vocabulário, sintaxe, significado e compreensão, podemos considerar que, embora estando numa fase inicial de validação, será de grande utilidade quer na investigação quer em contexto clínico.

\section{Referências}

Adams, M. J. (1990). Beginning to read: Thinking and learning about print. Cambridge, MA: MIT Press.

Allen, E., \& Rogers, L. (2008, November). Measuring reading comprehension in groups: A review of the Test of Silent Contextual Reading Fluency. Presented at the International Dyslexia Association Annual Convention. Seattle, WA. Retirado de 
https://www.proedinc.com/Downloads/TOSC RFMeasureReadCompGroups.pdf

Allen, E., Hammill, D. D., Rogers, L., \& Jagielko, J. (2010, March). Criterion-prediction validity of the Test of Silent Contextual Reading Fluency. Presented at the National Association of School Psychologists Annual Convention. Chicago, Illinois.

Alves, R. A., \& Castro, S. L. (2005). Despistagem da dislexia em adultos através do Questionário História de Leitura. IberPsicologia: Anales de la Revista de Psicologia General y Aplicada, 10 (8.9).

Barth, A. E., Catts, H. W., \& Anthony, J. L. (2009). The component skills underlying reading fluency in adolescent readers: A latent variable analysis. Reading and Writing, 22(5), 567-590.

Bell, S. M., McCallum, R. S., Kirk, E. R., Fuller, E. J., \& McCane-Bowling, S. (2007). Investigation of the psychometric attributes of the Test of Silent Contextual Reading Fluency. Assessment for Effective Intervention, 33(1), 39-46.

Berninger, V. W., Abbott, R. D., Trivedi, P., Olson, E., Gould, L., Hiramatsu, S., \& Scuilli Boyd, A. (2010). Applying the multiple dimensions of reading fluency to assessment and instruction. Journal of Psychoeducational Assessment, 28(1), 3-18.

Carvalho, A., \& Pereira, M. (2009). O Rei - Um teste para avaliação da fluência e precisão da leitura no $1^{\circ}$ e $2^{\circ}$ ciclos do Ensino Básico. Psychologica, 51, 283-305.

Chall, J. S. (1996). Stages of reading development (2nd ed.). Fort Worth: Harcourt Brace College Publishers.

Chard, D. J., Vaughn, S., \& Tyler, B. J. (2002). A synthesis of research on effective interventions for building reading fluency with elementary students with learning disabilities. Journal of Learning Disabilities, 35(5), 386-406. doi:10.1177/00222194020350050101

Denton, C. A., Barth, A. E., Fletcher, J. M., Wexler, J., Vaughn, S., Cirino, P. T., \& Francis, D. J. ( 2011 ). The relations among oral and silent reading fluency and comprehension in middle school: Implications for identification and instruction of students with reading difficulties. Scientific Studies of Reading, 15 (2), 109-135. doi:10.1080/10888431003623546

Ehri, L. C. (2005). Learning to read words: Theory, findings, and issues. Scientific Studies of Reading, 9(2), 167-188.

Fernandes, T., Araújo, S., Sucena, A., Reis, A., \& Castro, S. L. (2017). The 1-min Screening Test for reading problems in college students: Psychometric properties of the 1-min TIL. Dyslexia, 23(1), 66-87. doi:10.1002/dys.1548

Fernandes, S., Querido, L., Verhaeghe, A., \& Araújo, L. (2018). What is the relationship between reading prosody and reading comprehension in European Portuguese? Evidence from grades 2 to 5. Journal of Research in Reading. doi:10.1111/1467-9817.12248

Fernandes, S., Querido, L., Verhaeghe, A., Marques, C., \& Araújo, L. (2017). Reading development in European Portuguese: relationships between oral reading fluency, vocabulary and reading comprehension. Reading and Writing, 30(9), 1987-2007. doi:10.1007/s11145-017-9763-Z

Fernandes, S., Simões, C., Querido, L., \& Verhaeghe, A. (2015). Fluência na leitura oral de texto e de palavras: Estudo transversal com adolescentes portugueses. Revista Iberoamericana de Diagnóstico y Evaluación - e Avaliação Psicológica, 39(1), 113-124. Retirado de https://www.aidep.org/sites/default/files/201711/Art10.pdf

Fernández-Huerta, J. (1959). Medidas sencillas de lecturabilidad (simple readability measures). Consigna, 214, 29-32.

Fuchs, L. S., Fuchs, D., Hosp, M. K., \& Jenkins, J. R. (2001). Oral reading fluency as an indicator of reading competence: A theoretical, empirical, and historical analysis. Scientific Studies of Reading, 5, 239-256. doi:10.1207/S1532799XSSR0503_3.

Gagliano, A., Ciuffo, M., Ingrassia, M., Ghidoni, E., Angelini, D., Benedetto, L., ... \& Stella, G. (2015). Silent reading fluency: Implications for the assessment of adults with developmental dyslexia. Journal of Clinical and Experimental Neuropsychology,37(9), 972-980. doi:10.1080/13803395.2015.1072498 
Guy, J. (2005). Grandes civilizações: Egípcios; gregos; romanos; chineses; vikings; incas; astecas. Lisboa: Dinalivro.

Hammill, D. D., Wiederholt, J. L., \& Allen, E. A. (2006). Test of silent contextual reading fluency. Austin, TX: PRO-ED.

Hiebert, E. H., Samuels, S. J., \& Rasinski, T. (2012). Comprehension-based silent reading rates: What do we know? What do we need to know? Literacy Research and Instruction, 51(2), 110-124. doi: 10.1080/19388071.2010.531887

Hopkins, W. G. (2002, June 23). A scale of magnitudes for the effect statistics. In A new view of statistics. Retirado de http://www.sportsci.org/resource/stats/effectm ag.html

Hudson, R. F., Pullen, P. C., Lane, H. B., \& Torgesen, J. K. (2009). The complex nature of reading fluency: A multidimensional view. Reading \& Writing Quarterly, 25, 4-32. doi:10.1080/ 10573560802491208.

Jenkins, J. R., Fuchs, L. S., van den Broek, P., Espin, C., \& Deno, S. L. (2003). Sources of individual differences in reading comprehension and reading fluency. Journal of Educational Psychology, 95, 719-729. doi:10.1037/0022-0663.95.4.719.

Kim,Y.-S. , Wagner, R.K., \& Foster, E. (2011). Relations among oral reading fluency, silent reading fluency, and reading comprehension: A latent variables study of first-grade readers. Scientific Studies of Reading, 15(4), 338-362. doi:10.1080/10888438.2010.493964

Kuhn, M., Schwanenflugel, P. J., \& Meisinger, E. B. (2010). Aligning theory and assessment of reading fluency: Automaticity, prosody, and the definitions of fluency. Reading Research Quarterly, 45, 230-251. doi:10.1598/RRQ.45.2.4.

Kuhn, M. R., \& Stahl, S. (2003). Fluency: A review of developmental and remedial strategies. The Journal of Educational Psychology, 95, 3-22.

LaBerge, D., \& Samuels, S. J. (1974). Toward a theory of automatic information processing in reading. Cognitive Psychology, 6, 293-323. doi:10.1016/0010-0285(74)90015-2.

Lefly, D. L., \& Pennington, B. F. (2000). Reliability and validity of the adult reading history questionnaire. Journal of Learning Disabilities, 33(3), 286-296. doi:10.1177/002221940003300306

Nergård-Nilssen, T., \& Hulme, C. (2014). Developmental dyslexia in adults: Behavioural manifestations and cognitive correlates. Dyslexia, 20(3), 191-207. doi:10.1002/dys. 1477

OECD (2016). PISA 2015 Results (Volume I): Excellence and Equity in Education. PISA, OECD Publishing, Paris. doi: 10.1787/9789264266490-en

Paige, D.D., Rasinski, T.V., \& Magpuri-Lavell, T. (2012). Is fluent, expressive reading important for high school readers? Journal of Adolescent \& Adult Literacy, 56(1), 67-76.

Perfetti, C. A. (1985). Reading ability. New York, NY: Oxford University Press.

Piacente, T. (2012). Alfabetización inicial y alfabetización académica. Investigación y Evaluación de los procesos implicados en el aprendizaje del lenguaje escrito. Revista Iberoamericana de Diagnóstico y Evaluación - e Avaliação Psicológica, 33(1), 9-30. Retirado de http://www.aidep.org/03_ridep/R33/r33art1.p df

Pikulski, J. J., \& Chard, D. J. (2005). Fluency: Bridge between decoding and reading comprehension. The Reading Teacher, 58(6), 510-519. doi:10.1598/RT.58.6.2

Rasinski, T.V. (2006). Reading fluency instruction: Moving beyond accuracy, automaticity, and prosody. The Reading Teacher, 59(7), 704-706. doi:10.1598/RT.59.7.10

Rasinski, T.V. (2009). Essential Readings on Fluency. Newark, DE: International Reading Association.

Rasinski, T. V., Padak, N., Linek, W., \& Sturtevant, E. (1994). Effects of fluency development on urban second-grade readers. The Journal of Educational Research, 87(3), 158-165. doi:10.1080/00220671.1994.9941237

Rasinski, T. V., Padak, N. D., McKeon, C. A., Wilfong, L. G., Friedauer, J. A., \& Heim, P. (2005). Is reading fluency a key for successful high school reading? Journal of Adolescent \& Adult Literacy, 49(1), 22-27. doi:10.1598/JAAL.49.1.3

Samuels, S. J. (2007). The DIBELS tests: Is speed of barking at print what we mean by reading fluency? 
Seok, S., \& DaCosta, B. (2014). Oral reading fluency as a predictor of silent reading fluency at secondary and postsecondary levels. Journal of Adolescent \& Adult Literacy, 58(2), 157-166. doi:10.1002/jaal.342

Tamboer, P., \& Vorst, H. (2015). A new SelfReport Inventory of Dyslexia for students: Criterion and construct validity. Dyslexia, 21(1), 1-34. doi:10.1002/dys.1492

Trainin, G., \& Swanson, H. L. (2005). Cognition, metacognition, and achievement of college students with learning disabilities. Learning Disability Quarterly, 28(4), 261-272. doi: $10.2307 / 4126965$

Tops, W., Callens, M., Lammertyn, J., Van Hees, V., \& Brysbaert, M. (2012). Identifying students with dyslexia in higher education. Annals of Dyslexia, 62(3), 186-203. doi:10.1007/s11881-012-0072-6

Warmington, M., Stothard, S. E., \& Snowling, M. J. (2013). Assessing dyslexia in higher education: The York adult assessment batteryrevised. Journal of Research in Special Educational Needs, 13(1), 48-56. doi:10.1111/j.1471-3802.2012.01264.x

Yildirim, K., \& Ates, S. (2012). Silent and oral reading fluency: Which one is the best predictor of reading comprehension of Turkish elementary students. International Journal on New Trends in Education and Their Implications, 3(4), 79-91. Recuperado de http://www.ijonte.org/FileUpload/ks63207/Fil e/07.yildirim.pdf 